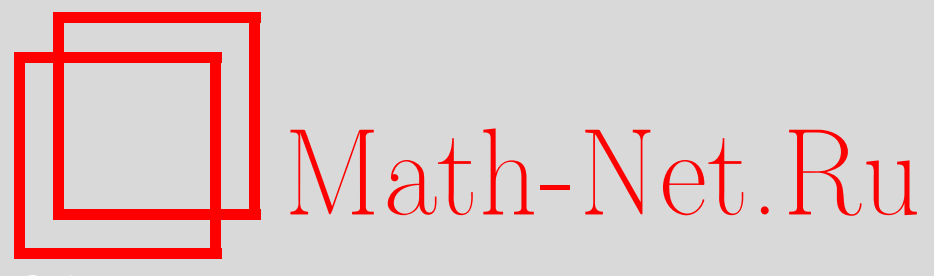

В. М. Федоров, Представление комплексных банаховых пространств пространством непрерывных функций на компакте, Матем. заметки, 2013, том 93, выпуск 2, 316320

DOI: https://doi.org/10.4213/mzm9103

Использование Общероссийского математического портала Math-Net.Ru подразумевает, что вы прочитали и согласны с пользовательским соглашением http://www . mathnet.ru/rus/agreement

Параметры загрузки:

IP : 3.89 .185 .249

26 апреля 2023 г., 14:57:43

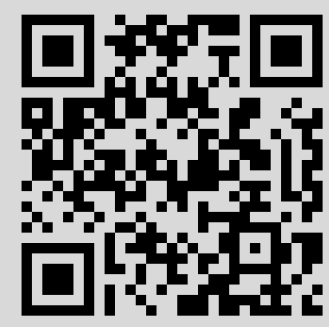




\section{Представление комплексных банаховых пространств пространством непрерывных функций на компакте}

\section{В. М. Федоров}

Существует много различных представлений пространства $C(X)$ непрерывных функций на компакте $X$. Результаты, характеризующие его среди действительных банаховых пространств при помощи естественных свойств нормированного пространства, получены Аренсом-Келли, Джерисоном и Майерсом. В настоящей работе дается характеристика $C(X)$ среди комплексных банаховых пространств. Для этого вначале устанавливаются свойства функционалов Майерса на комплексном нормированном пространстве $E$ и определяются представления пространства орбитальных функций $C_{\sigma}(X)$ относительно действия однопараметрической группы гомеоморфизмов $X$. Изложение ведется одновременно для действительного и комплексного случая.

Пусть $E$ - нормированное пространство над полем действительных или комплексных чисел с единичным шаром $S$, а $E^{\prime}$ - его сопряженное пространство с единичным шаром $S^{\prime}$. Обозначим через $\mathfrak{W}=\mathfrak{W}(E)$ совокупность всех максимальных конусов $K \subset E$, на которых норма аддитивна, а через $\mathfrak{V}=\mathfrak{V}\left(E^{\prime}\right)$ - соответствующее множество минимальных граней $\Gamma \subset S^{\prime}$ таких, что каждому максимальному конусу $K \in \mathfrak{W}$ соответствует минимальная грань $\Gamma_{K} \in \mathfrak{V}$ по формуле

$$
\Gamma_{K} \doteqdot\left\{\alpha \in S^{\prime} \mid \operatorname{Re} \alpha(x)=\|x\|, x \in K\right\} .
$$

Заметим, что всякий максимальный конус $K \in \mathfrak{W}$ совпадает с некоторым конусом вида

$$
K_{\alpha} \doteqdot\{x \in E \mid \operatorname{Re} \alpha(x)=\|\alpha\|\}, \quad \alpha \in S^{\prime} .
$$

Рассмотрим линейную оболочку $L \doteqdot \mathrm{sp}_{\mathbb{R}}(K)$ как упорядоченное пространство с положительным конусом $K \in \mathfrak{W}$. Нетрудно заметить, что функция $\Delta_{x}(y) \doteqdot\|x+y\|-\|y\|$ по переменной $y \in K$ является невозрастающей, поэтому при всех $x \in E$ существует предел по направлению $K$

$$
\varphi_{K}(x) \doteqdot \lim _{y \in K} \Delta_{x}(y)=\inf _{y \in K} \Delta_{x}(y),
$$

Функция $\varphi_{K}(x)$, заданная при всех $x \in E$, называется функционалом Майерса, ассоциированным с максимальным конусом $K \in \mathfrak{W}$ (см. [1; с. 133], [2; с. 148]).

ПредложениЕ 1. Функционал Майерса $\varphi_{K}(x), K \in \mathfrak{W}$, определенный на нормированном пространстве Е, обладает следующими свойствами:

a) $-\|x\| \leqslant \varphi_{K}(x) \leqslant\|x\|$ для всех $x \in E$;

b) $\varphi_{K}(x)= \pm\|x\|$ тогда и только тогда, когда $x \in \pm K$;

c) $\varphi_{K}(\lambda x)=\lambda \varphi_{K}(x)$ при всех $\lambda \in \mathbb{R}_{+} u x \in E$;

d) $\varphi_{K_{1}}(x)=\varphi_{K_{2}}(x)$ при всех $x \in E$ тогда и только тогда, когда $K_{1}=K_{2}$;

е) $\varphi_{K}(x+y) \leqslant \varphi_{K}(x)+\varphi_{K}(y)$ при всех $x, y \in E$; функционал $\varphi_{K}(x)$ является непрерывной субнормой на пространстве $Е$ и линейным функиионалом над полем действительных чисел $\mathbb{R}$ на линейной оболочке $L$ конуса $K$;

f) функционал $\varphi_{K}(x)$ является линейным на пространстве $E$ над полем действительных чисел $\mathbb{R}$ тогда и только тогда, когда функиия $\mathrm{p}(x)=\|x\|$, заданная при всех $x \in K$, имеет единственное продолжение на $E$ до линейного функционала с нормой единица;

Работа выполнена при поддержке Российского фонда фундаментальных исследований (грант № 12-01-00169).

DOI: $10.4213 / \operatorname{mzm} 9103$ 
g) если $\Gamma_{K} \in \mathfrak{V}$ есть минимальная грань шара $S^{\prime} \subset E^{\prime}$, ассоциированная с максимальным конусом $K \in \mathfrak{W}$, то функиионал Майерса равен $\varphi_{K}(x) \doteqdot \sup _{\alpha \in \Gamma_{K}} \operatorname{Re} \alpha(x)$ при всех $x \in E$.

Из этих свойств нетрудно заметить, что функционал $\varphi_{K}(x)$ является линейным над $\mathbb{R}$ тогда и только тогда, когда он является нечетным, т.е. выполняется равенство $\varphi_{K}(-x)=$ $-\varphi_{K}(x)$ при всех $x \in E$. В этом случае он имеет единственное линейное продолжение из подпространства $L$ на все пространство $E$ с сохранением нормы, равной 1 , и минимальная грань $\Gamma_{K}$ шара $S^{\prime}$, соответствующая максимальному конусу $K \in \mathfrak{W}$, состоит из одной точки.

Обозначим через $C(X)$ пространство всех непрерывных и ограниченных функций $f$ : $X \rightarrow \mathbb{F}$ со значениями в поле $\mathbb{F}$ действительных или комплексных чисел, определенных на хаусдорфовом топологическом пространстве $X$ с чебышевской нормой $\|f\|=\sup _{x \in X}|f(x)|$.

Пусть $\delta: X \rightarrow C^{\prime}(X)$ является отображением в сопряженное пространство $C^{\prime}(X)$, где $\delta(x)=\delta_{x}$ функционал Дирака, равный $\delta_{x}(f)=f(x)$ при всех $f \in C(X)$. Тогда отображение $\delta$ непрерывно в слабой* топологии пространства $C^{\prime}(X)$ и является гомеоморфизмом между $X$ и $\delta(X)$ тогда и только тогда, когда пространство $X$ вполне регулярно [3; c. 158-161]. Если $X$ вполне регулярно, то слабое* замыкание образа $\delta(X)$ состоит из всех тех крайних точек $\alpha$ единичного шара в $C^{\prime}(X)$, для которых выполняется равенство $\operatorname{Re} \alpha(1)=1[2 ;$ с. 146]. Далее всюду будем предполагать, что пространство $X$ является вполне регулярным.

Если $X$ компактно, то множество крайних точек единичного шара в $C^{\prime}(X)$ слабо* замкнуто. Конус $K \subset C(X)$, на котором норма аддитивна, тогда и только тогда является максимальным в пространстве $C(X)$, когда он принимает следующий вид:

$$
K_{t, x}=\left\{f \in C(X) \mid \operatorname{Re} e^{i t} f(x)=\|f\|\right\}, \quad x \in X, \quad t \in \mathbb{R} .
$$

Таким образом, в этом случае всякая минимальная грань $\Gamma_{K}$ единичного шара в $C^{\prime}(X)$ является крайней точкой, а ее действительная часть является функционалом Майерса $\varphi_{K}$.

Обозначим далее через $\sigma=\left\{\sigma_{t} \mid t \in \mathbb{T}\right\}$ непрерывную однопараметрическую группу гомеоморфизмов $\sigma_{t}: X \rightarrow X$ пространства $X$, для которой каждая точка $x \in X$ имеет период $2 \pi$ за исключением, быть может, неподвижных точек группы $\sigma$, т.е. $\sigma(x)=x$. При этом в случае поля действительных чисел $\mathbb{F}=\mathbb{R}$ параметр $t \in \mathbb{T} \doteqdot \pi \mathbb{Z}$ считается дискретным, а в случае поля комплексных чисел $\mathbb{F}=\mathbb{C}$ параметр $t \in \mathbb{T} \doteqdot \mathbb{R}$ считается непрерывным.

Через $C_{\sigma}(X)$ обозначается подпространство в $C(X)$ всех орбитальных функиий группы $\sigma$, т.е. таких функций, что $f\left(\sigma_{t} x\right)=e^{i t} f(x)$ при всех $(t, x) \in \mathbb{T} \times X$. Например, если $X=S^{\prime}$ является единичным шаром сопряженного пространства $E^{\prime}$, в котором введена слабая* топология, то действие однопараметрической группы $\sigma=\left\{\sigma_{t} \mid t \in \mathbb{T}\right\}$ гомеоморфизмов $X$ определяется естественным образом по формуле $\sigma_{t}(\alpha)=e^{i t} \alpha$ при всех $(t, \alpha) \in \mathbb{T} \times X$.

Так как отображение $\delta$ коммутирует с элементами группы $\sigma$, то единичный шар $S_{\sigma}^{\prime} \subset$ $C_{\sigma}^{\prime}(X)$ является слабо* замкнутой выпуклой оболочкой множества $\delta(X)[4 ;$ с. 478]. Совокупность крайних точек $\operatorname{ex}\left(S_{\sigma}^{\prime}\right)$ содержится в слабом* замыкании образа $\delta(X)$. Множество $\delta(X)$ является слабо* замкнутым в шаре $S_{\sigma}^{\prime}$ в том и только в том случае, когда пространство $X$ компактно. Таким образом, если $X$ компактно, то множество крайних точек $\operatorname{ex}\left(S_{\sigma}^{\prime}\right)$ и множество максимальных конусов пространства $C_{\sigma}(X)$ находится во взаимно однозначном соответствии с теми точками $x \in X$, которые имеют период $2 \pi$, т.е. $\sigma(x) \neq x$. При этом каждый максимальный конус $K \subset C_{\sigma}(X)$, на котором норма аддитивна, определяется уравнением $\operatorname{Re} v(x)=\|v\|$ в некоторой фиксированной точке $x \in X$, где $\sigma(x) \neq x$.

ОПРедЕЛЕНиЕ 1. Подпространство $M \subset N$ нормированного пространства $N \subset C(X)$ называется отделяющим (вполне регулярным) подпространством в $N$ [2; с. 150], если какова бы не была точка $x_{0} \in X$ и замкнутое множество $A \subset X$, не содержащее $x_{0} \notin A$, для 
которых существует функция $u \in N$, удовлетворяющая неравенству

$$
\operatorname{Re} u\left(x_{0}\right)>\sup _{x \in A}|u(x)| \quad\left(\|u\|=\operatorname{Re} u\left(x_{0}\right)>\sup _{x \in A}|u(x)|\right),
$$

существует функция $v \in M$, также удовлетворяющая этому неравенству:

$$
\operatorname{Re} v\left(x_{0}\right)>\sup _{x \in A}|v(x)| \quad\left(\|v\|=\operatorname{Re} v\left(x_{0}\right)>\sup _{x \in A}|v(x)|\right) .
$$

Всякое нормированное пространство $E$ над полем действительных или комплексных чисел допускает изометричное отображение на отделяющее подпространство в $C_{\sigma}(X)$ на вполне регулярном пространстве $X$ с непрерывной однопараметрической группой $\sigma$ гомеоморфизмов, где в качестве $X$ можно взять совокупность всех крайних точек минимальных граней единичного шара $S^{\prime} \subset E^{\prime}$ сопряженного пространства $E^{\prime}$, наделенного слабой* топологией.

Если $M \subset C_{\sigma}(X)$ вполне регулярно, то всякий конус вида $K_{x}=\{v \in M \mid \operatorname{Re} v(x)=\|v\|\}$, где $\sigma x \neq x$, на котором норма аддитивна, является максимальным конусом в подпространстве $M$, т.е. $K_{x} \in \mathfrak{W}(M)$. При этом функционал Майерса $\varphi_{K_{x}}$ на подпространстве $M$, ассоциированный с максимальным конусом $K_{x}$, принимает вид $\varphi_{K_{x}}(v)=\operatorname{Re} v(x)$ при всех $v \in M$. Если, кроме того, пространство $X$ компактно, то каждый максимальный конус $K \in \mathfrak{W}(M)$, на котором норма аддитивна, принимает вид $K=K_{x}$ при некотором $x \in X$ и $\sigma x \neq x$.

Теорема 1. Нормированное пространство Е над полем действительных или комплексных чисел тогда и толъко тогда изометрично вполне регулярному подпространству в $C_{\sigma}(X)$ на вполне регулярном $X$ с непрерывной однопараметрической группой $\sigma$ гомеоморфизмов, когда существует такое множество $Y \subset \operatorname{ex}\left(S^{\prime}\right)$ крайних точек единичного uара $S^{\prime} \subset E^{\prime}$, что

а) каждый элемент $Y$ является одноточечной минимальной гранъю $S^{\prime}$;

b) множество $Y$ инвариантно $\sigma(Y)=Y$ относительно действия группы $\sigma$;

c) слабо* замкнутая выпуклая оболочка множества $Y$ равна $\overline{\mathrm{co}}(Y)=S^{\prime}$.

Если, кроме того, $X$ компактно, то эти условия равносильны одному из следующих условий:

d) каждый функиионал Майерса является линейным над полем действительных чисел и множество всех функционалов Майерса $Y=\left\{\varphi_{K} \mid K \in \mathfrak{W}\right\}$ (или множество $Y \cup 0)$ является слабо* компактным в сопряженном пространстве $E^{\prime}$;

е) каждая минимальная грань единичного шара $S^{\prime}$ является крайней точкой и множество ех $\left(S^{\prime}\right)$ всех крайних точек этого шара (или множество ех $\left.\left(S^{\prime}\right) \cup 0\right)$ является слабо* компактным в пространстве $E^{\prime}$.

В случае компактного пространства $X$ утверждение этой теоремы вытекает из следующих соображений. Пусть $I: E \rightarrow C_{\sigma}(X)$ является изометричным отображением на вполне регулярное подпространство $M=I(E)$. Так как пересечение с шаром $S_{\sigma}^{\prime}$ прообраза $I^{\prime-1}(A)$ любого экстремального подмножества $A \subset S^{\prime}$ при сопряженном отображении $I^{\prime}: C_{\sigma}^{\prime}(X) \rightarrow E^{\prime}$ является экстремальным подмножеством шара $S_{\sigma}^{\prime}$, то в силу теоремы Крейна-Мильмана каждая крайняя точка единичного шара $S^{\prime}$ является образом некоторой крайней точки единичного шара $S_{\sigma}^{\prime}$, т.е. имеют место следующие включения: $\operatorname{ex}\left(S^{\prime}\right) \subset I^{\prime}\left(\operatorname{ex}\left(S_{\sigma}^{\prime}\right)\right) \subset I^{\prime}(\delta(X))$.

С другой стороны, в силу первой части теоремы 1 каждый функционал вида $\alpha_{x}=I^{\prime} \delta_{x}$, где $\sigma(x) \neq x$, является одноточечной минимальной гранью шара $S^{\prime}$ и его действительная часть совпадает с функционалом Майерса $\varphi_{K_{\alpha_{x}}}=\operatorname{Re} \alpha_{x}$, где $K_{\alpha_{x}}$ является максимальным конусом $E$, на котором норма аддитивна. Отсюда будут справедливы следующие равенства:

$$
\left\{\alpha_{x}=I^{\prime} \delta_{x} \mid \sigma(x) \neq x\right\}=\left\{\alpha \mid \operatorname{Re} \alpha=\varphi_{K}, K \in \mathfrak{W}\right\} \quad \text { и } \quad\left\{I^{\prime} \delta_{x} \mid \sigma(x)=x\right\}=\{0\} .
$$


Таким образом, если группа $\sigma$ не имеет неподвижных точек, то множество $X$ гомеоморфно множеству $\operatorname{ex}\left(S^{\prime}\right)$, а если группа $\sigma$ имеет неподвижные точки, то $X$ при отождествлении всех неподвижных точек будет гомеоморфно множеству $\operatorname{ex}\left(S^{\prime}\right) \cup 0$, т.е. имеют место (d) и (е). Обратно, если выполнено одно из этих условий (d) или (e), то в силу первой части образ канонического отображения $J: E \rightarrow C_{\sigma}(X)$ является вполне регулярным подпространством в $C_{\sigma}(X)$, где $X$ обозначает слабо* компактное множество, $\operatorname{paвное~} \operatorname{ex}\left(S^{\prime}\right)$ или $\operatorname{ex}\left(S^{\prime}\right) \cup 0$.

В действительном случае Аренс-Келли указали два критерия изометричности пространству $C(X)$ на компакте $X$ в терминах нормированного пространства. Мы укажем несколько эквивалентных критериев для пространства $C(X)$ комплекснозначных непрерывных функций.

Предложение 2. Пусть $E$ - банахово пространство и множество $Y$ состоит из всех крайних точек минимальных граней сопряженного шара $S^{\prime}$. Тогда пространство $E$ в том и только в том случае изометрично пространству $C_{\sigma}(X)$ на некотором хаусдорфовом компакте $X$ с непрерывной однопараметрической группой $\sigma$ гомеоморфизмов, когда выполнено одно из следующих условий:

а) для каждой системы максимальных конусов $\left\{K_{\alpha}\right\}_{\alpha \in A} \subset \mathfrak{W}$, для которых пересечение $\bigcap_{\alpha \in A} K_{\alpha}=0$ равно нулю, найдутся такие сети $\left\{\alpha_{n}^{\prime}\right\},\left\{\alpha_{n}^{\prime \prime}\right\} \subset A$ и число $t \neq$ $\pi(\bmod 2 \pi)$, чmo $\lim \left\{\varphi_{K_{\alpha_{n}^{\prime}}}(v)+\varphi_{K_{\alpha_{n}^{\prime \prime}}}\left(e^{i t} v\right)\right\}=0$ nри всех $v \in E ;$

b) для каждого подмножества $A \subset Y$, для которого при всех $t \neq 0(\bmod 2 \pi)$ nересечение $\bar{A} \cap \sigma_{t}(\bar{A})=\varnothing$ слабых* замыканий множеств $A$ и $\sigma_{t}(A)$ является пустым множеством, пересечение соответствующих максималъных конусов $\bigcap_{\alpha \in A} K_{\alpha} \neq 0$ не равно нулю;

с) для каждого подмножества $A \subset Y$, для которого при всех $t \neq 0(\bmod 2 \pi)$ пересечение $\bar{A} \cap \sigma_{t}(\bar{A})=\varnothing$ слабых* замыканий множеств $A$ и $\sigma_{t}(A)$ является пустым множеством, существует такой ненулевой элемент $е \in E$, что $\operatorname{Re} \alpha(e)=\|e\| n p u$ всех $\alpha \in A$;

d) пространство Е канонически изометрично пространству $C_{\sigma}(X)$ на слабо* компактном множестве $X=\bar{Y}$, совпадающим со слабым* замыканием множества $Y$ в сопряженном пространстве $E^{\prime}$.

ОПРеДЕЛЕниЕ 2. Говорят [2; с. 154], что банахово пространство $E$ обладает свойством (A), если для каждой системы максимальных выпуклых подмножеств $\left\{V_{\alpha}\right\}_{\alpha \in A} \subset \partial S$ границы единичного шара $\partial S \subset E$, у которых пересечение $\bigcap_{\alpha \in A} V_{\alpha}=\varnothing$ пусто, существуют такие сети $\left\{\alpha_{n}^{\prime}\right\},\left\{\alpha_{n}^{\prime \prime}\right\} \subset A$ и число $t \neq \pi(\bmod 2 \pi)$, что $\lim \left\{\rho\left(u, V_{\alpha_{n}^{\prime}}\right)+\lim \rho\left(e^{i t} u, V_{\alpha_{n}^{\prime \prime}}\right)\right\}=2$ при всех $u \in E$, где $\rho(u, V)=\inf _{v \in V}\|u-v\|$ обозначает расстояние от точки $u \in E$ до множества $V \subset E$.

Для того чтобы банахово пространство $E$ обладало свойством (А), необходимо и достаточно, чтобы выполнялось одно из эквивалентных условий предложения 2 . В случае, если элемент $e \in \operatorname{ex}(S)$ является крайней точкой, то каждый элемент $v \in E$ принадлежит некоторому множеству вида $U_{t} \doteqdot\left\{u \in E \mid\left\|u+e^{i t} e\right\|=\|u\|+1\right\}, t \in \mathbb{T}$. При этом все элементы максимального конуса $K \in \mathfrak{W}$ принадлежат одному из этих множеств $U_{t}$ и для всякого максимального конуса $K \in \mathfrak{W}$ существует такое единственное число $t \in \mathbb{T}(\bmod 2 \pi)$, что $e^{i t} e \in K$.

Теорема 2. Банахово пространство Е над полем $\mathbb{F}$ действительных или комплексных чисел в том и только том случае изометрически изоморфно пространству непрерывных функиий $C(X)$ на некотором хаусдорфовом компакте $X$, когда его единичный шар $S \subset E$ имеет крайнюю точку е $\in \operatorname{ex}(S)$ и выполнено одно из эквивалентных условий предложения 2 или свойство (A). 
В самом деле, всякая непрерывная функция $f \in C(X)$, модуль которой равен $|f(x)|=1$ при всех $x \in X$, является крайней точкой единичного шара в $C(X)$. Поскольку пространство $C(X)$ изометрично пространству $C_{\sigma}\left(e^{i \mathbb{T}} \times X\right)$, где $\sigma_{\tau}\left(e^{i t}, x\right)=\left(e^{i(\tau+t)}, x\right)$ при всех $\tau$, $t \in \mathbb{T}$ и $x \in X$, то имеют место все эквивалентные условия предложения 2 , а следовательно, и свойство $(\mathrm{A})$.

С другой стороны, если единичный шар $S$ в пространстве $E$ имеет крайнюю точку $e \in \operatorname{ex}(S)$ и выполнено каждое из эквивалентных условий предложения 2 , то $E$ канонически изометрично пространству $C_{\sigma}(X)$, где $X$ является слабым* замыканием множества крайних точек единичного шара $S^{\prime} \subset E^{\prime}$. В силу теоремы 1 и указанного выше замечания для каждой крайней точки $\alpha \in \operatorname{ex}\left(S^{\prime}\right)$ найдется такое $t \in \mathbb{T}$, что $\operatorname{Re} e^{i t} \alpha(e)=1$ и, следовательно, $\alpha$ принадлежит одному из непересекающихся слабо* замкнутых множеств $X_{t} \doteqdot\left\{\alpha \in X \mid \operatorname{Re} e^{i t} \alpha(e)=1\right\}$. Так как имеют место равенства $\sigma_{\tau} X_{t}=X_{\tau+t}$ и $\bigcup_{t \in \mathbb{T}} X_{t}=X$, то пространство $C_{\sigma}(X)$ изометрично пространству $C\left(X_{0}\right)$ на слабо* компактном фактормножестве $X_{0}=X / \sigma$.

В действительном случае свойство (А) было введено Аренсом и Келли [5] и была доказана его необходимость, как это сформулировано в теореме 2. Утверждения предложения 2 в действительном случае и достаточность теоремы 2 в действительном случае принадлежат Джерисону [6]. Указанные выше теоремы можно применить к специальному классу банаховых пространств, подробно изученных в действительном случае Акиловым [7], [8], Нахбином [9], Гуднером [10] и Келли [11], для доказательства соответствующих результатов над полем комплексных чисел.

\section{СПИСОК ЦИТИРОВАННОЙ ЛИТЕРАТУРЫ}

[1] S. B. Myers, Ann. of Math. (2), 49:1 (1948), 132-140. [2] М. М. Дей, Нормированные линейные пространства, Библиотека сборника "Математика", ИЛ, М., 1961. [3] Дж. Л. Келли, Общая топология, Наука, М., 1981. [4] Н. Данфорд, Дж. Шварц, Линейные операторы. Т. 1. Общая теория, ИЛ, М., 1962. [5] R. F. Arens, J. L. Kelley, Trans. Amer. Math. Soc., 62 (1947), 499-508. [6] M. Jerison, Trans. Amer. Math. Soc., 70 (1951), 103-113. [7] Г. П. Акилов, ДАН СССР, 57 (1947), 643-646. [8] Г. П. Акилов, ДАН СССР, 59 (1948), 417-418. [9] L. Nachbin, Trans. Amer. Math. Soc., 68 (1950), 28-46. [10] D. B. Goodner, Trans. Amer. Math. Soc., 69 (1950), 89-108. [11] J. L. Kelley, Trans. Amer. Math. Soc., 72 (1952), 323-326.

\section{В. М. Федоров}

Поступило

Московский государственный университет

30.03.2011

им. М. В. Ломоносова

E-mail: vferdorov@rambler.ru 